\title{
HER2-Low Status and Response to Neoadjuvant Chemotherapy in HER2 Negative Early Breast Cancer
}

Luciana de Moura Leite ( $\square$ luciana.leite@accamargo.org.br)

A.C. Camargo Cancer Center https://orcid.org/0000-0003-4620-081X

Marcelle Goldner Cesca

A.C. Camargo Cancer Center

Monique Celeste Tavares

A.C. Camargo Cancer Center

Debora Maciel Santana

A.C. Camargo Cancer Center

Erick Figueiredo Saldanha

A.C. Camargo Cancer Center

Paula Tavares Guimarães

A.C. Camargo Cancer Center

Daniella Dias Silva Sá

A.C. Camargo Cancer Center

Maria Fernanda Evangelista Simões

A.C. Camargo Cancer Center

Rafael Lima Viana

A.C. Camargo Cancer Center

Francisca Giselle Rocha

A.C. Camargo Cancer Center

Simone Klog Loose

A.C. Camargo Cancer Center

Sinara Figueiredo Silva

A.C. Camargo Cancer Center

Rafaela Pirolli

A.C. Camargo Cancer Center

Camilla Albina Zanco Fogassa

A.C. Camargo Cancer Center

Bruna Raphaeli Silva Mattos

A.C. Camargo Cancer Center

Fernando Augusto Batista Campos 
A.C. Camargo Cancer Center

\section{Solange Moraes Sanches}

A.C. Camargo Cancer Center

\section{Vladmir Cláudio Cordeiro de Lima}

A.C. Camargo Cancer Center

Noam Falbel Pondé

A.C. Camargo Cancer Center

\section{Research Article}

Keywords: HER2, HER2-low breast cancer, antibody-drug conjugates, trastuzumab-deruxtecan, trastuzumab-duocarmazine

Posted Date: April 26th, 2021

DOl: https://doi.org/10.21203/rs.3.rs-420934/v1

License: (c) (i) This work is licensed under a Creative Commons Attribution 4.0 International License. Read Full License

Version of Record: A version of this preprint was published at Breast Cancer Research and Treatment on August 18th, 2021. See the published version at https://doi.org/10.1007/s10549-021-06365-7. 


\title{
HER2-low status and response to neoadjuvant chemotherapy in HER2 negative early breast cancer
}

\begin{abstract}
Purpose

Recently, phase I studies with novel antibody drug conjugates targeting HER2 suggested benefit in HER2-low patients - defined as immunohistochemistry(IHC) +1 or +2 FISH/ISH non-amplified, with advanced breast cancer(BC). Data on the prognostic value of HER2-low in early stage disease is scarce. The purpose of this study was to evaluate the impact of HER2-low status on response to neoadjuvant chemotherapy(NACT) and survival outcomes in early stage HER2negative $B C$
\end{abstract}

\section{Methods}

Records from all BC patients treated with NACT from January 2007 to December 2018 in a single cancer center were retrospectively reviewed. Primary objective was to compare differences between pathologic complete response $(p C R)$ and relapse free survival(RFS) in luminal HER2-low/HER2-0 and triple negative(TNBC) HER2-low/HER2-0.

\section{Results}

855 non-HER2-positive patients were identified. Median follow-up was 59 months. 542 had luminal BC (63.4\%) and 313 TNBC (36.6\%). 285 (33.3\%) were HER2-low. Among luminal tumors, 145 had HER2 IHC+1 (26.8\%) and 91 IHC+2/ISH non-amplified (16.8\%). In TNBC, only 36 had HER2 IHC+1 (11.5\%) 
and $13 \mathrm{IHC}+2 / \mathrm{ISH}$ non-amplified (4.2\%). Among luminal/HER2-low and luminal/HER2-0 population, there was a high proportion of clinical T3/4 $(61.5 \%$ vs $69.2 \%, p=0.053)$, node positive $(74.2 \%$ vs $66.3 \%, p=0.27)$ and stage III tumors (63.1\% vs $65 \%, p=0.51)$. The same was true TNBC/HER-low as compared to TNBC/HER2-0, despite a non-statistically significant higher cT4 among TNBC/HER-low (32.7\% vs. 19.3\%, $\mathrm{p}=0.17)$. pCR was $13 \%$ in luminal/HER2-low versus $9.5 \%$ in luminal/HER2-0 $(p=0.27)$, and 51\% in TNBC/HER2-low versus $47 \%$ in TNBC/HER2-0 ( $p=0.64)$. 5y RFS was $72.1 \%$ in luminal/HER2-low and $71.7 \%$ in luminal/HER2-0 $(p=0.47)$, and $75.6 \%$ in TNBC/HER2-low versus $70.8 \%$ in TNBC/HER2-0 ( $p=0.23)$. HER2-low status was not associated with RFS in multivariate analysis (HR $0.83,95 \% \mathrm{Cl} 0.6-1.11, \mathrm{p}=0.21)$.

\section{Conclusion}

Our data does not support HER2-low as a biologically distinct BC subtype, with no predictive effect on $\mathrm{pCR}$ after NACT nor prognostic value on survival outcomes.

\section{Introduction}

HER2-positive status has for more than 20 years defined a subgroup of breast cancer (BC) patients who benefit from anti-HER2 therapy [1, 2]. Beyond that, HER2 status defines a distinct BC subtype with aggressive biological behavior and historically worse prognosis, a reality that was changed after the incorporation of HER2-therapy [3-5]. Patients with HER2-low status - defined as 
immunohistochemistry $(\mathrm{IHC})+1$ or $+2 / \mathrm{ISH}$ non-amplified, have not, however, benefitted from the use of either trastuzumab nor pertuzumab [6-8].

Recently, the results of phase I studies with novel antibody drug conjugates (ADC) targeting HER2 (trastuzumab-deruxtecan and trastuzumabduocarmazine) have suggested a level of efficacy in HER2-low patients with advanced breast cancer, with objective response rates ranging between 32 to $37 \%$ in a heavily pretreated population $[9,10]$. This led to the hypothesis that HER2-low tumors might represent a separate disease subset, distinct from other luminal and triple negative (TNBC) cancers, with distinct clinical properties and thus the potential for new targeted therapies. Indeed, several trials are currently exploring the potential of anti-HER2 agents in HER2-low patients.

Little is known about HER2-low early BC and data on its prognosis is scarce [11]. Besides, its response to standard chemotherapy has not been previously reported. More data on this patient population is urgently needed in order to guide the integration of these new drugs into treatment guidelines.

The majority of large phase 3 trials conducted in the past involving luminal or triple negative BC did not collect detailed HER2 status information, making retrospective cohorts particularly valuable in this scenario. Therefore, the purpose of this study was to evaluate the impact of HER2-low status on response to neoadjuvant chemotherapy (NACT) and survival outcomes in early stage HER2-negative BC.

\section{Methods}

\subsection{Study Design and Participants}


This is a retrospective cohort which enrolled all early breast cancer patients treated with neoadjuvant chemotherapy from January 2007 to December 2018 in a single cancer center in South America (A. C. Camargo Cancer Center, São Paulo, Brazil). Patients had to meet the following inclusion criteria: age $\geq 18$ years old, histologically proven invasive carcinoma of the breast, clinical stage I to III, treatment with neoadjuvant chemotherapy followed by surgery with curative intent. Patients with HER2 positive BC - IHC +3 or IHC +2 FISH/ISH amplified, or patients with IHC +2 FISH/ISH equivocal, were excluded. Other exclusion criteria included: insufficient data about tumor pathological characteristics or treatment received, history of synchronous or metachronous breast cancer, and diagnosis of other synchronous invasive cancer.

Subtype was defined by IHC for hormonal receptors (HR) and HER2, and/or FISH when HER2 staining was +2 , on pre-treatment biopsy specimens. Tumors were defined as luminal if estrogen $(E R)$ and/or progesterone $(\mathrm{PgR})$ receptors were $\geq 1 \%$, or TNBC, if ER $<1 \%$ and $\mathrm{PgR}<1 \%$. HER2-low status was defined as HER2 IHC 1+ or 2+/ISH non-amplified. Other patients were classified as HER2-0. HER2 was accessed using standard antibodies and ISH-techniques and classified according to the ASCO/CAP guidelines available during the different time periods [12-14]. Pathological review of specimens was not performed.

The CONSORT diagram for the study is shown on Supplement figure 1. A total of 1.495 consecutive BC patients were screened, 303 HER2 positive and 2 HER2 $+2 /$ ISH equivocal were excluded. Other 337 patients were excluded for other reasons. The following baseline clinicopathological characteristics were collected from medical records: age at diagnosis, gender, menopausal status, histological subtype and grade, Ki67, TNM staging and anatomic stage groupings. We also 
collected data regarding treatment offered (chemotherapy, surgery and radiotherapy) and sites of first disease recurrence. This study was approved by the institutional Internal Ethics Review Board.

\section{$\underline{1.3 \text { Statistical Analysis }}$}

The primary objective of this study was to investigate whether HER2-low status impacts efficacy outcomes, including pathologic complete response ( $p C R)$ and relapse free survival (RFS) for patients with luminal/TNBC undergoing neoadjuvant chemotherapy. pCR was defined as no invasive carcinoma in the breast and in axillary lymph nodes at the time of surgery. RFS was defined as the time from breast cancer diagnosis to locoregional or contralateral relapse, distant metastasis, or death (from any cause), whichever occurred first.

Secondary objectives included to describe the demographics and clinicalpathological characteristics of this population and to assess the impact of low HER2 expression on overall survival (OS) and patterns of recurrence.

The clinical-pathological and demographical variables were represented as relative and absolute frequencies. Fisher's Exact test and Mann-Whitney's test were used to explore differences in categorial and continuous variables between luminal HER2-0/HER2-low and TNBC HER2-0/HER2-low patients.

Survival curves were generated using the Kaplan-Meier method and were compared using the log-rank test. Tests were considered statistically significant when p-values (two-tailed) <0.05. Baseline clinicopathological and demographical variables associated with OS or RFS with Wald`s p-value $\leq 0,2$ in 
the univariate analysis were selected for multivariate analysis. All statistical analyses were performed with software SPSS 23.0 (SPSS, Chicago, IL).

\section{Results}

\section{Population and treatment patterns}

Between January 2007 and December 2018, 855 eligible non-HER2-positive patients were identified. Median follow-up time for all cohort was 59 months. There were 2 male and 853 female patients. 542 patients had luminal BC $(63.4 \%)$ and 313 TNBC (36.6\%). The distribution of HER2-0, HER2 IHC+1 and HER2 IHC+2/ISH non-amplified in this cohort was $570(66.7 \%), 181(21.2 \%)$ and 104 (12.2\%), respectively, corresponding to a total 285 (33.3\%) HER2-low patients. Among luminal tumors, 145 patients had HER2 IHC +1 (26.8\%) and $91 \mathrm{IHC}$ +2/ISH non-amplified (16.8\%). This proportion was lower in TNBC, with only 36 patients with HER2 IHC +1 (11.5\%) and 13 with IHC +2/ISH non-amplified (4.2\%).

The baseline characteristics of this population and the patterns of treatment offered, stratified by subtype and the presence or absence of HER2-low status, are described in Table 1. Most patients were premenopausal, had invasive ductal carcinomas and grade II/III tumors, irrespective of subtype or HER2-low status. Among the luminal subtype tumors, there was a high proportion of clinical T3/4, node positive and stage III tumors, with no difference between the luminal/HER2low and the luminal/HER2-0 population. Among TNBC tumors, the same was true despite a non-statistically significant larger number of more advanced tumors among TNBC/HER-low as compared to TNBC/HER2-0. Median Ki67 was higher for TNBC as compared to luminal BC, irrespective of HER2 status. 
Anthracyclines plus taxanes was the preferred chemotherapy regimen, with a higher proportion of dose dense regimens and neoadjuvant carboplatin being used in TNBC/HER2-low and TNBC/HER2-0 (Table 1). Mastectomy plus axillary dissection was performed in more than $70 \%$ of luminal/HER2-low and luminal/HER2-0 patients. TNBC/HER2-0 patients had slightly less mastectomies when compared to the rest of the population, probably reflecting the higher proportion of clinical T2 tumors and clinically negative axillae. Most patients in this cohort received adjuvant radiotherapy.

When comparing only HER2-low versus HER2-0 in the whole cohort, the vast majority of HER2-low patients had luminal subtype (82.8\%), with a slightly greater proportion of cT4 $(34.7 \%$ vs $27.5 \%, p=0.06)$ and a significantly higher proportion of clinical node positive tumors ( $74 \%$ vs $65.4 \%, p=0.01)$, whereas HER2-0 patients were more evenly distributed between luminal (53.7\%) and TNBC (46.3\%), with a greater proportion of grade III and high Ki67 (Supplementary Table 1).

\section{Efficacy outcomes}

Pathological complete response was achieved in $13 \%$ of the luminal luminal/HER2-low versus $9.5 \%$ of the luminal/HER2-0 patients $(p=0.27)$, and in $51 \%$ of TNBC/HER2-low versus $47 \%$ of TNBC/HER2-0 $(p=0.64)$, suggesting no impact of HER2-low status on pCR (Figure 1) after anthracycline-based neoadjuvant chemotherapy.

Five-years RFS and OS were also largely unaffected. Luminal/HER2-low patients had a $5 y$ RFS of $72.1 \%$ versus $71.7 \%$ for luminal/HER2-0 patients $(p=0.47)$, and a $5 y$ OS of $89.4 \%$ versus $83.8 \%(p=0.11)$, respectively (Figures $2 A$ and $C$ ). TNBC/HER2-low patients had a $5 y$ RFS of $75.6 \%$ versus $70.8 \%$ in TNBC/HER2- 
$0(p=0.23)$, and a $5 y$ OS of $79.1 \%$ versus $80.3 \%(p=0.71)$, respectively (Figures 2B and D).

HER2-low status was not associated with RFS both in univariate and multivariate analysis ( $\mathrm{HR} 0.83,95 \% \mathrm{Cl} 0.6-1.11, \mathrm{p}=0.21)$, as show in Table 2. In univariate analysis for OS, HER2-low status was associated with improved OS, but this association was lost after adjusting for other factors in the multivariate model (Table 3). Survival curves considering the whole population stratified by HER2-0, HER2 +1 and HER2 +2 or HER2-0/HER2-low follow the same behavior and are shown in supplementary figure 2 . Sites of recurrence - bone, visceral and central nervous system (CNS) - were not significantly different among HER2-low patients, although CNS and visceral recurrences occurred in a greater proportion among the HER2-0 population (Supplementary figure 3).

\section{Discussion}

In this cohort, 33.3\% of patients were classified as HER2-low, a somewhat lower frequency than observed in previous reports where the prevalence of this emerging subtype ranged between $40-50 \%[15,16]$. This discrepancy seems to be largely driven by the lower proportion of HER2 +1 in this cohort $(21.2 \%)$, when compared with other series, were most of the HER2-low patients were indeed classified as HER2 +1 [17]. One possible explanation would be that, the distinction between HER2-0 and HER2 +1 until recently had no clinical implications, which may impact the precision with which pathologists evaluate low scores. Indeed, previously published data showed that for cases considered as HER2-0 by local pathologists, there was only $15 \%$ concordance after central review, with as many as $76 \%$ being reclassified to HER2 +1 [18]. 
Concordant with more recent data, the vast majority of HER2-low patients $(82.8 \%)$ had hormone receptor (HR) positive breast cancer [17]. However, differently from other publications [17, 19], HER2-low status did not imply more advanced tumors among luminal BC, with only slightly more T4 tumors among TNBC. When looking at the entire cohort there were more node positive cases among HER2-low, but this was possibly biased given that the majority of this population comprised luminal patients for which neoadjuvant chemotherapy is commonly indicated in locally advance disease.

Regarding the HER2-low status impact on survival outcomes, it initially looked like HER2 +2 led to better survival, but this did not hold true when adjusting for disease subtype and other prognostic factors on multivariate analysis. The prognostic role of HER2-low status remains controversial, with some retrospective studies suggesting worse prognosis for HER2-low in localized node positive and luminal early $\mathrm{BC}$, although these relatively older works made comparisons only between HER2-0/HER2 +1 versus HER2 +2/ISH negative [16, 19].

A recent paper, however, that used the current HER2-low definition, did not find a prognostic impact for HER2-low status on overall survival in a large cohort of 3.689 patients with predominantly advanced BC [17]. Interestingly, in this paper, comprehensive molecular analysis were performed using PAM50 and individual gene expression data, and the authors were able to differentiate HRpositive/HER2-low BC as a more distinct biologic entity pertaining to Luminal A intrinsic subtype with a higher ERBB2 expression level versus HRpositive/HER2-0 BC, a group that encompassed a larger proportion of Luminal B and Basal-like subtypes with lower ERBB2 expression. This was not observed 
for TNBC that was composed predominantly of Basal-like subtype and had low levels of ERBB2 expression regardless of being HER2-low or not. The underlying biological intrinsic subtypes might explain why in our cohort, although not significant, more CNS and visceral recurrences were seen in the HER2-0 population, which was possibly enriched for the Basal-like subtype.

Not surprisingly, HER2-low status did not predict for response to standard chemotherapy in this cohort. A similar finding was preliminarily presented at San Antonio 2020, in a retrospective series of 331 patients, with no difference in the pCR rates between HR-positive HER2-0 and HER2-low subgroups (8\% vs $13 \%$, $p=0.35$ ), but a higher $p C R$ rate, although non-statistically significant, among TNBC HER2-0 versus HER2-low tumors ( $56 \%$ vs. $39 \%, p=0.09$ ) [20].

Although the activity of new ADCs is being extensively studied in advanced HER2-low BC, clinical trials testing theses agents in the early stage setting are lacking [21]. In this scenario, the only data presented was the adjuvant trial of the HER2 targeting vaccine, Nenelipepimut-S, associated with Trastuzumab with negative results $[22,23]$.

Our study has several limitations, including relatively small sample size, its retrospective nature, absence of central pathological review and patient inclusion across a large time period, during which different guidelines for HER2 testing and interpretation were in use. Still it sheds some light on the clinical and biological behavior of this large BC population which usually accounts for young individuals with locally advanced HR-positive, who have very poor pCR rates following NACT and therefore could largely benefit from the incorporation of new treatment approaches. 
For the abovementioned subset new classes of drugs, like CDK4/6 inhibitors, have not improved outcome [24], and more importantly than for the TNBC subgroup, for which the field is rapidly moving towards neoadjuvant chemoimmunotherapy with outstanding $\mathrm{pCR}$ rates [25], it represents an area of unmet clinical need. Addressing this issue NCT04553770, is now recruiting patients with HR-positive HER2-low early BC to neoadjuvant trastuzumab-deruxtecan with or without anastrozole with the primary end point of $\mathrm{pCR}$.

In conclusion, although our data does not support HER2-low as a biologically distinct $\mathrm{BC}$ subtype, it may reflect a specially interesting population in which to test new HER2-targeting ADC. Therefore, efforts should be made to better identify those patients in real life settings and to better determine how to incorporate these drugs into (neo)adjuvant treatment plan.

Table 1 - Baseline demographics and clinical-pathological characteristics stratified by subtype and HER2-low status ( $\mathrm{N}=855)$.

\begin{tabular}{|c|c|c|c|c|c|c|}
\hline \multirow[t]{2}{*}{ Characteristics } & \multicolumn{3}{|c|}{ Luminal (N 542) } & \multicolumn{3}{|c|}{ TNBC (N 313) } \\
\hline & $\begin{array}{l}\text { HER2-Iow } \\
(\mathrm{N}=236)\end{array}$ & $\begin{array}{l}\text { HER2-0 } \\
(\mathrm{N}=306)\end{array}$ & $\mathbf{p}^{\mathbf{a}}$ & $\begin{array}{l}\text { HER2-Iow } \\
(\mathrm{N}=49)\end{array}$ & $\begin{array}{l}\text { HER2-0 } \\
(\mathrm{N}=264)\end{array}$ & $p^{a}$ \\
\hline $\begin{array}{l}\text { Median age (range) } \\
\text { Menopausal status ( }\end{array}$ & 46 (24-77) & $46(26-82)$ & 0.44 & $44(26-84)$ & $44(24-90)$ & 0.71 \\
\hline Premenopausal & $150(63.6)$ & $196(64.1)$ & 0.61 & $29(59.2)$ & $164(62.1)$ & 0.56 \\
\hline Postmenopausal & 80 (33.9) & $98(32)$ & & $19(38.8)$ & $86(32.6)$ & \\
\hline Perimenopausal & $5(2.1)$ & $11(3.6)$ & & $1(2)$ & $14(5.3)$ & \\
\hline Male & $1(0.4)$ & $1(0.3)$ & & 0 & 0 & \\
\hline
\end{tabular}




\section{Histology (\%)}

Ductal

Lobular

Other

missing

cT (\%)

T1

T2

T3

T4

cN (\%)

NO

N1

N2

N3

Clinical Stage (\%)

I

II

III

\section{Histologic Grade (\%)}

II

III

missing

Ki67

Median

(IQR)

Missing(\%)

\section{Chemotherapy (\%)}

Anthracycline+taxane

Dose dense ${ }^{b}$

Carboplatin

Radiotherapy (\%)

Surgery (\%)

Mastectomy

Breast conserving

Bilateral mastectomy

Axillary dissection

$$
\begin{array}{cc}
204(86.4) & 246(80.4) \\
17(7.2) & 40(13.1) \\
15(6.4) & 20(6.5) \\
0 & 0
\end{array}
$$

9 (3.8)

4 (1.3)

$82(34.7) \quad 90(29.5)$

62 (26.3) 106 (34.6)

$83(35.2) \quad 106$ (34.6)

0.053

3 (6.1)

12 (4.5)

0.17

20 (40.8)

126 (47.8)

10 (20.4)

75 (28.4)

16 (32.7)

51 (19.3)

0.27

13 (26.5)

94 (35.6)

0.19

21 (42.9)

111 (42)

7 (14.3)

40 (15.2)

8 (16.3)

19 (7.2)

0.51

1 (2)

10 (3.8)

0.65

$\begin{array}{cc}87(36.9) & 105(34.3) \\ 149(63.1) & 199(65)\end{array}$

21 (42.9)

128 (48.5)

27 (55.1)

126 (47.7)

$\begin{array}{cc}26(11) & 21(6.9) \\ 135(57.2) & 190(62.1) \\ 67(28.4) & 89(29.1) \\ 8(3.4) & 6(1.9)\end{array}$

0.20

0

13 (26.5)

3 (1.1)

0.91

33 (67.3)

65 (24.6)

3 (6.2)

179 (67.8)

17 (6.5)

0.36

70

(60-90)

80

0.80

$\begin{array}{ll}(25-60) & (25-70) \\ 12(5.1) & 24(7.8)\end{array}$

4 (8.2)

(60-90)

15 (5.7)

$\begin{array}{cc}229(97) & 295(96.4) \\ 56(23.7) & 93(30.4) \\ 7(3) & 11(3.6)\end{array}$

224 (94.9) $296(96.7)$

0.81

0.10

0.81

0.38

0.97

$\begin{array}{cc}182(77.1) & 238(77) \\ 52(22) & 69(22.4) \\ 2(0.9) & 2(0.6)\end{array}$

$173(73.3) \quad 222(72.5)$
0.92

30 (61.2)

$\begin{array}{cc}49(100) & 255(96.6) \\ 23(46.9) & 142(53.8) \\ 23(46.9) & 140(53) \\ 49(100) & 249(94.3) \\ 35(71.4) & 156(59.1) \\ 14(28.6) & 100(37.9) \\ 0 & 8(3)\end{array}$

$159(60.2)$
0.25

0.44

0.41

0.14

0.24

1.0 


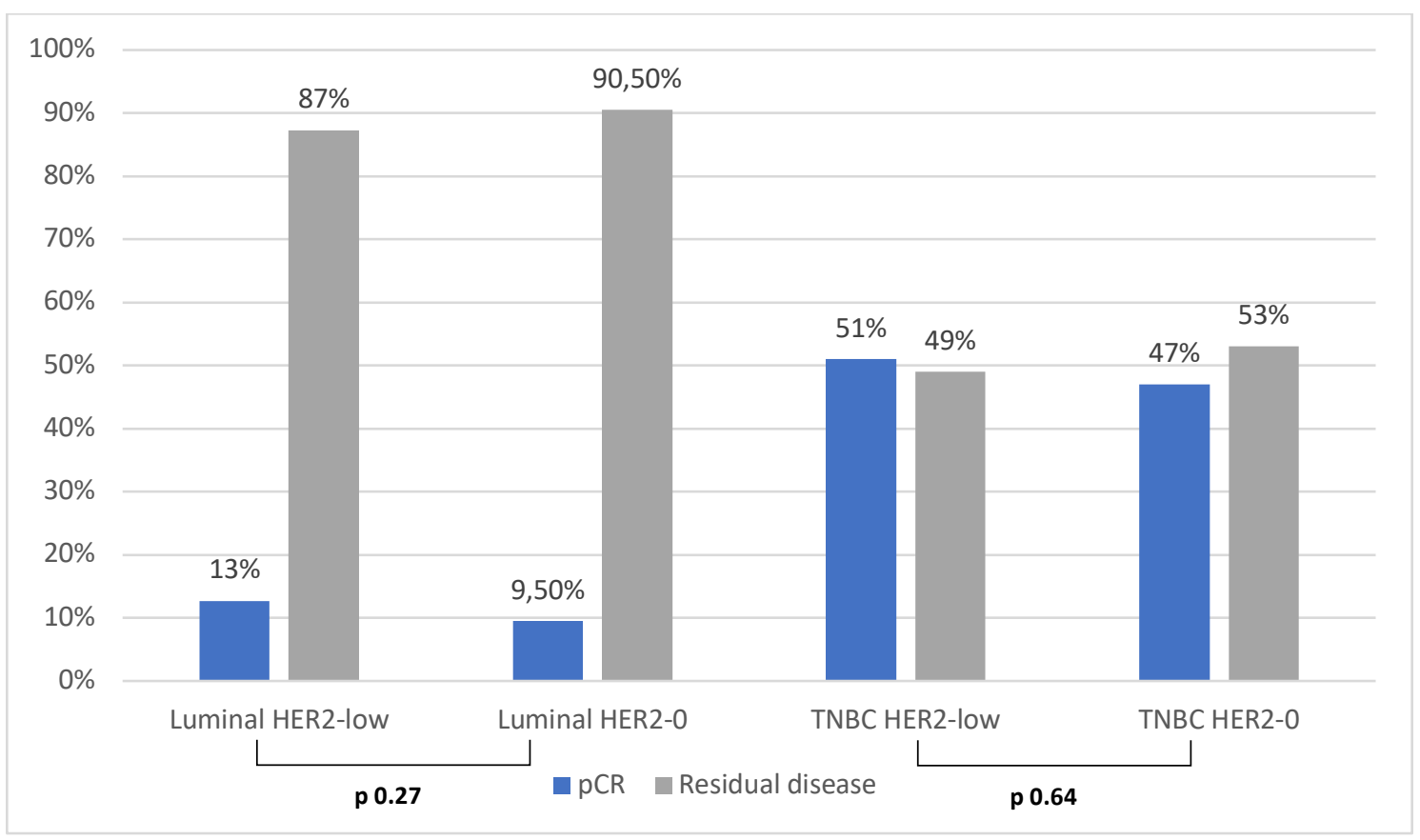

Figure 1. Frequency of $p C R$ in Luminal/HER2-low $(N=236)$, Luminal/HER2-0 $(N=306)$ and TNBC/HER2-low ( $\mathbf{N}=49)$, TNBC/HER2-0 ( $\mathbf{N = 2 6 4 ) . ~ p C R ~ w a s ~ d e f i n e d ~ a s ~ n o ~ i n v a s i v e ~}$ carcinoma in the breast and in axillary lymph nodes at the time of surgery. Frequencies were compared with Fisher's exact test.

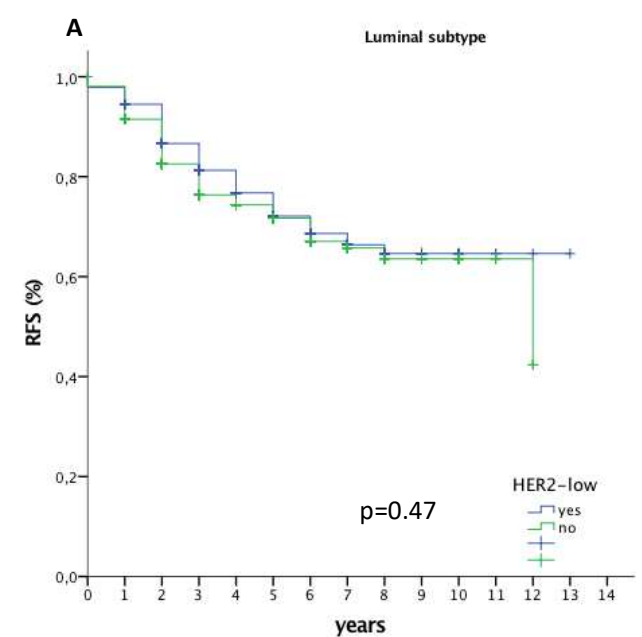

C

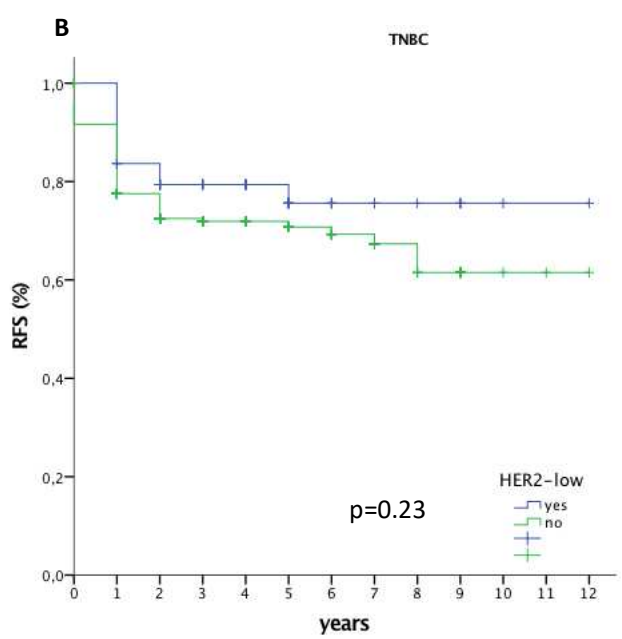

D 

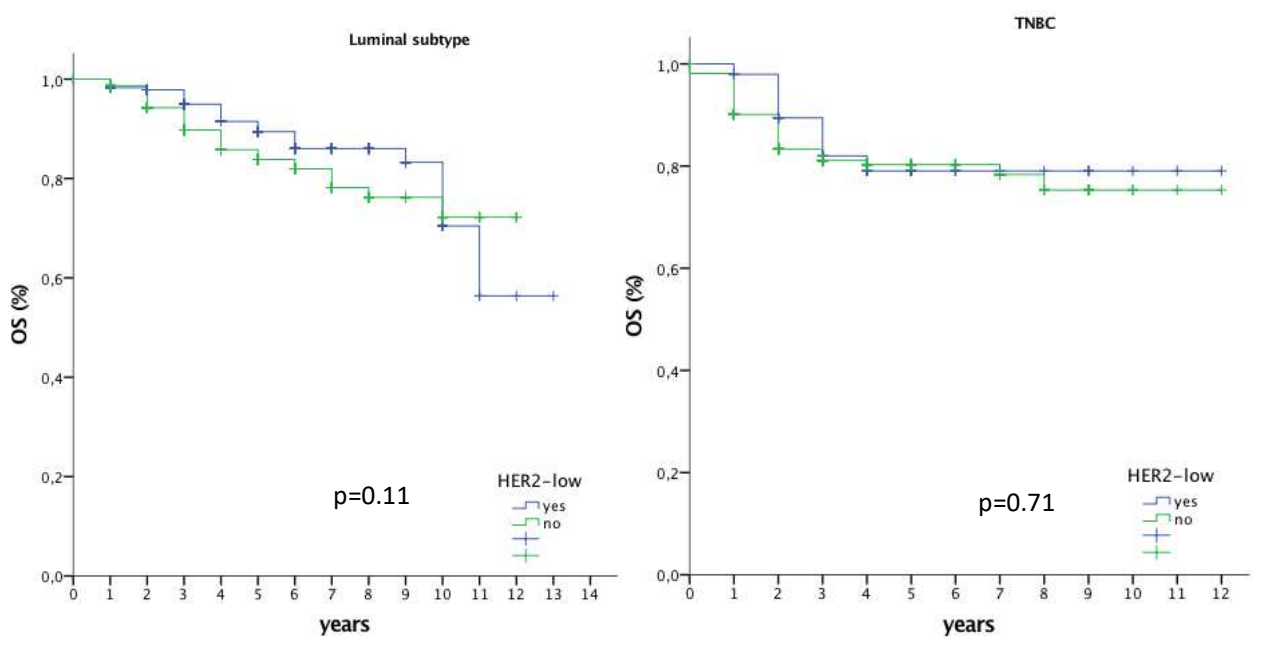

Figure 2. Relapse-free and overall survival curves according to HER2-low classification among molecular subtypes. A and B show relapse-free survival curves according to molecular subtype (luminal/TNBC) stratified by HER2-low status. $C$ and D show overall survival curves according to molecular subtype (luminal/TNBC) stratified by HER2-low status. Survival curves were estimated with the Kaplan-Meier method and compared with log-rank test.

Table 2 - Univariate and multivariate analysis for RFS in the entire cohort. Factors associated with RFS with $p \leq 0.2$ in the univariate analysis were included in the multivariate model.

\begin{tabular}{lcccc}
\hline Variable & \multicolumn{2}{c}{ Univariate analysis } & \multicolumn{2}{c}{ Multivariate analysis } \\
\hline & Hazard ratio $(95 \% \mathrm{Cl})$ & $\mathrm{p}$ & Hazard ratio $(95 \% \mathrm{Cl})$ & $\mathrm{p}$ \\
\hline HER2-low vs HER2-0 & $0.81(0.61-1.06)$ & 0.13 & $0.83(0.63-1.11)$ & 0.21 \\
pCR & $0.29(0.19-0.45)$ & $<0.0001$ & $0.22(0.14-0.35)$ & $<0.0001$ \\
cT3/4 vs T1/2 & $1.64(1.24-2.18)$ & 0.001 & $1.33(0.99-1.78)$ & 0.06 \\
cN positive & $1.76(1.29-2.40)$ & $<0.0001$ & $1.70(1.24-2.33)$ & 0.001 \\
TNBC vs Luminal & $1.12(0.98-1.27)$ & 0.11 & $1.42(1.23-1.63)$ & $<0.0001$ \\
Premenopausal vs & $0.96(0.74-1.25)$ & 0.74 & & \\
Peri/Postmenopausal & & & & \\
Histologic Grade II/III vs I & $1.22(0.71-2.09)$ & 0.49 & & \\
Ki67 $\geq 20 \%$ & $1.06(0.75-1.51)$ & 0.72 & & \\
\hline
\end{tabular}


Table 3 - Univariate and multivariate analysis for OS in the entire cohort. Factors associated with OS with $p \leq 0.2$ in the univariate analysis were included in the multivariate model.

\begin{tabular}{lcccc}
\hline Variable & \multicolumn{2}{c}{ Univariate analysis } & \multicolumn{2}{c}{ Multivariate analysis } \\
\hline & Hazard ratio $(95 \% \mathrm{Cl})$ & $\mathrm{p}$ & Hazard ratio $(95 \% \mathrm{Cl})$ & $\mathrm{p}$ \\
\hline HER2-low vs HER2-0 & $0.65(0.44-0.95)$ & 0.03 & $0.72(0.48-1.10)$ & 0.10 \\
pCR & $0.33(0.19-0.59)$ & $<0.0001$ & $0.23(0.12-0.41)$ & $<0.0001$ \\
cT3/4 vs T1/2 & $1.67(1.13-2.46)$ & 0.01 & $1.41(0.95-2.10)$ & 0.09 \\
cN positive & $1.85(1.21-2.83)$ & 0.004 & $1.76(1.16-2.74)$ & 0.009 \\
TNBC vs Luminal & $1.32(1.11-1.56)$ & 0.002 & $1.61(1.34-1.95)$ & $<0.0001$ \\
Premenopausal vs & $1.00(0.70-1.42)$ & 1.0 & & \\
Peri/Postmenopausal & & & & \\
Histologic Grade II/III vs I & $1.35(0.66-2.78)$ & 0.42 & & \\
Ki67 $\geq 20 \%$ & $1.39(0.85-2.27)$ & 0.19 & & \\
\hline
\end{tabular}

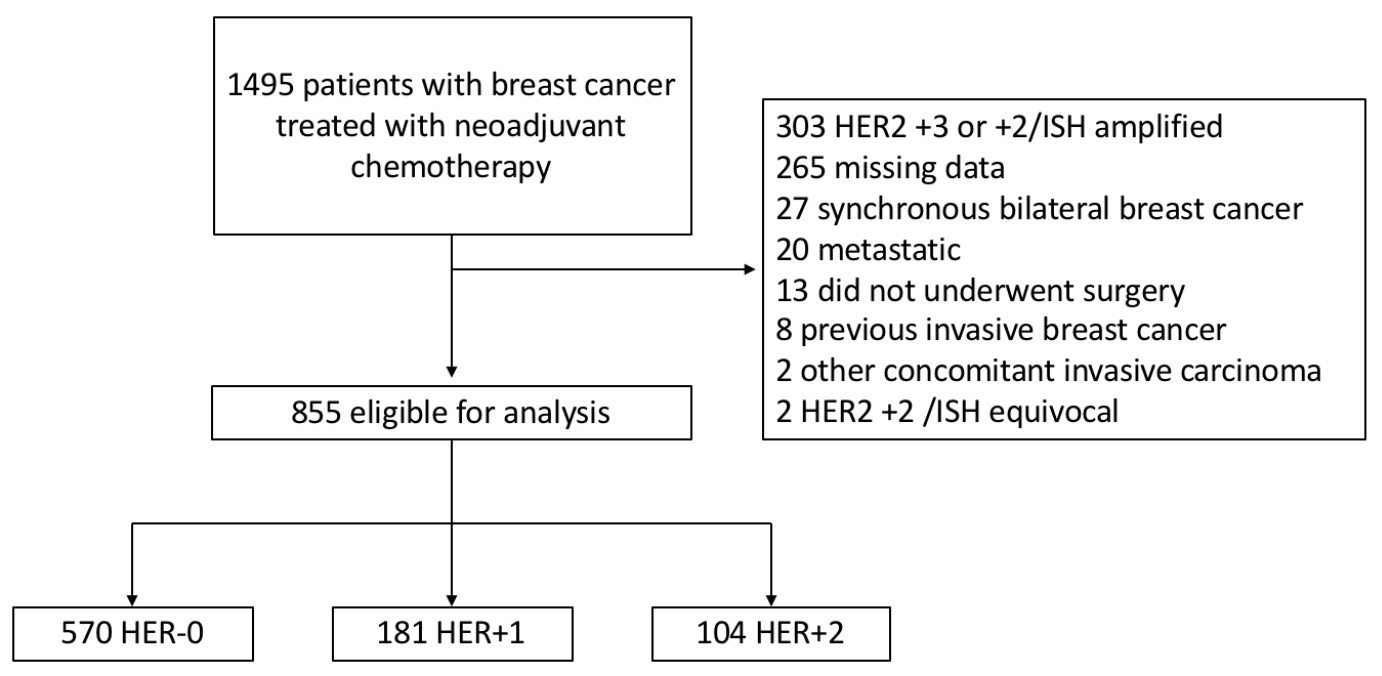

Supplementary figure 1 - CONSORT diagram for study entry. 
Supplementary Table 1 - Baseline characteristics stratified by HER2-low status.

\begin{tabular}{|c|c|c|c|}
\hline Characteristics & $\begin{array}{c}\text { HER2-Iow } \\
(\mathrm{N}=285)\end{array}$ & $\begin{array}{l}\text { HER2-0 } \\
(N=570)\end{array}$ & $\mathbf{p}^{a}$ \\
\hline Median age (range) & $46(24-84)$ & $45(24-90)$ & 0.15 \\
\hline \multicolumn{4}{|c|}{ Menopausal status (\%) } \\
\hline Premenopausal & $179(62.8)$ & $360(63.2)$ & 0.22 \\
\hline Postmenopausal & $99(34.7)$ & $184(32.3)$ & \\
\hline Perimenopausal & $6(2.1)$ & $25(4.4)$ & \\
\hline Male & $1(0.4)$ & $1(0.1)$ & \\
\hline \multicolumn{4}{|l|}{ Histology (\%) } \\
\hline Ductal & $246(86.3)$ & $472(82.8)$ & 1.0 \\
\hline Lobular & $18(6.3)$ & $49(8.6)$ & \\
\hline Other & $18(6.3)$ & $48(8.4)$ & \\
\hline missing & $3(1.1)$ & $1(0.2)$ & \\
\hline \multicolumn{4}{|l|}{ cT (\%) } \\
\hline T1 & $12(4.2)$ & $16(2.8)$ & 0.06 \\
\hline $\mathrm{T} 2$ & $102(35.8)$ & $216(37.9)$ & \\
\hline T3 & $72(25.3)$ & $181(31.8)$ & \\
\hline $\mathrm{T} 4$ & $99(34.7)$ & $157(27.5)$ & \\
\hline cN positive $(\%)$ & $211(74)$ & $373(65.4)$ & 0.01 \\
\hline \multicolumn{4}{|l|}{ Clinical Stage (\%) } \\
\hline I & $1(0.4)$ & $12(2.1)$ & 0.08 \\
\hline II & $108(37.9)$ & $233(40.9)$ & \\
\hline III & $176(61.7)$ & $325(57)$ & \\
\hline \multicolumn{4}{|l|}{ Histologic Grade (\%) } \\
\hline I & $26(9.1)$ & $24(4.2)$ & $<.001$ \\
\hline II & $148(51.9)$ & $255(44.7)$ & \\
\hline III & $100(35.1)$ & $268(47)$ & \\
\hline missing & $11(3.9)$ & $23(4.1)$ & \\
\hline Median Ki67 & 40 & 60 & $<.001$ \\
\hline (IQR) & $(25-70)$ & $(30-80)$ & \\
\hline Missing(\%) & $16(5.6)$ & $39(6.8)$ & \\
\hline \multicolumn{4}{|l|}{ Subtype (\%) } \\
\hline Luminal & $236(82.8)$ & $306(53.7)$ & $<.001$ \\
\hline TNBC & $49(17.2)$ & $264(46.3)$ & \\
\hline
\end{tabular}



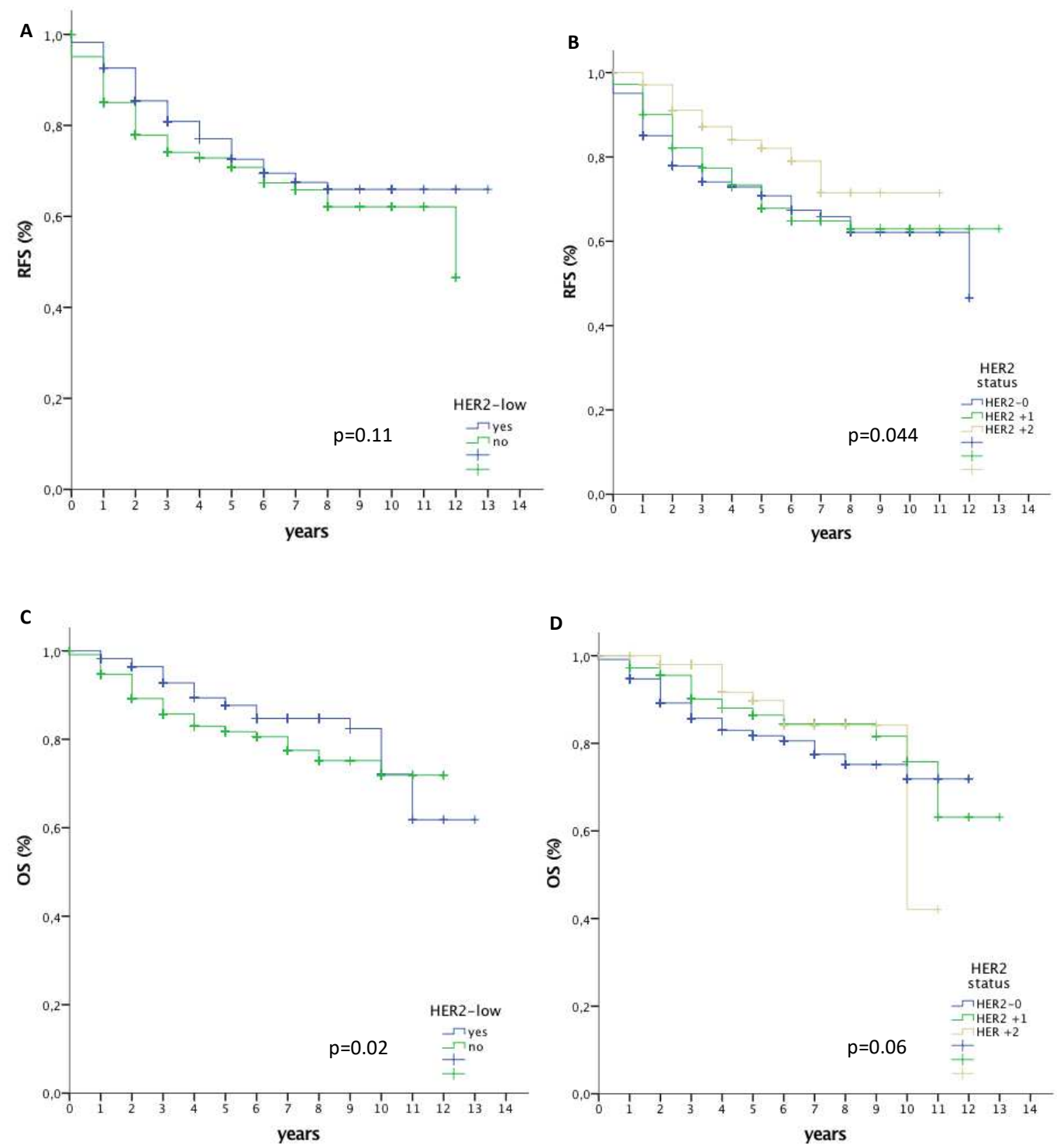

Supplementary figure 2. Relapse-free and overall survival curves according to HER2low classification in the entire cohort. $A$ and $B$ show RFS according to HER2-low status and HER2-0/HER2+1/HER2+2 for the whole cohort. $C$ and D show OS according to HER2-low status and HER2-0/HER2+1/HER2+2 for the whole cohort. 

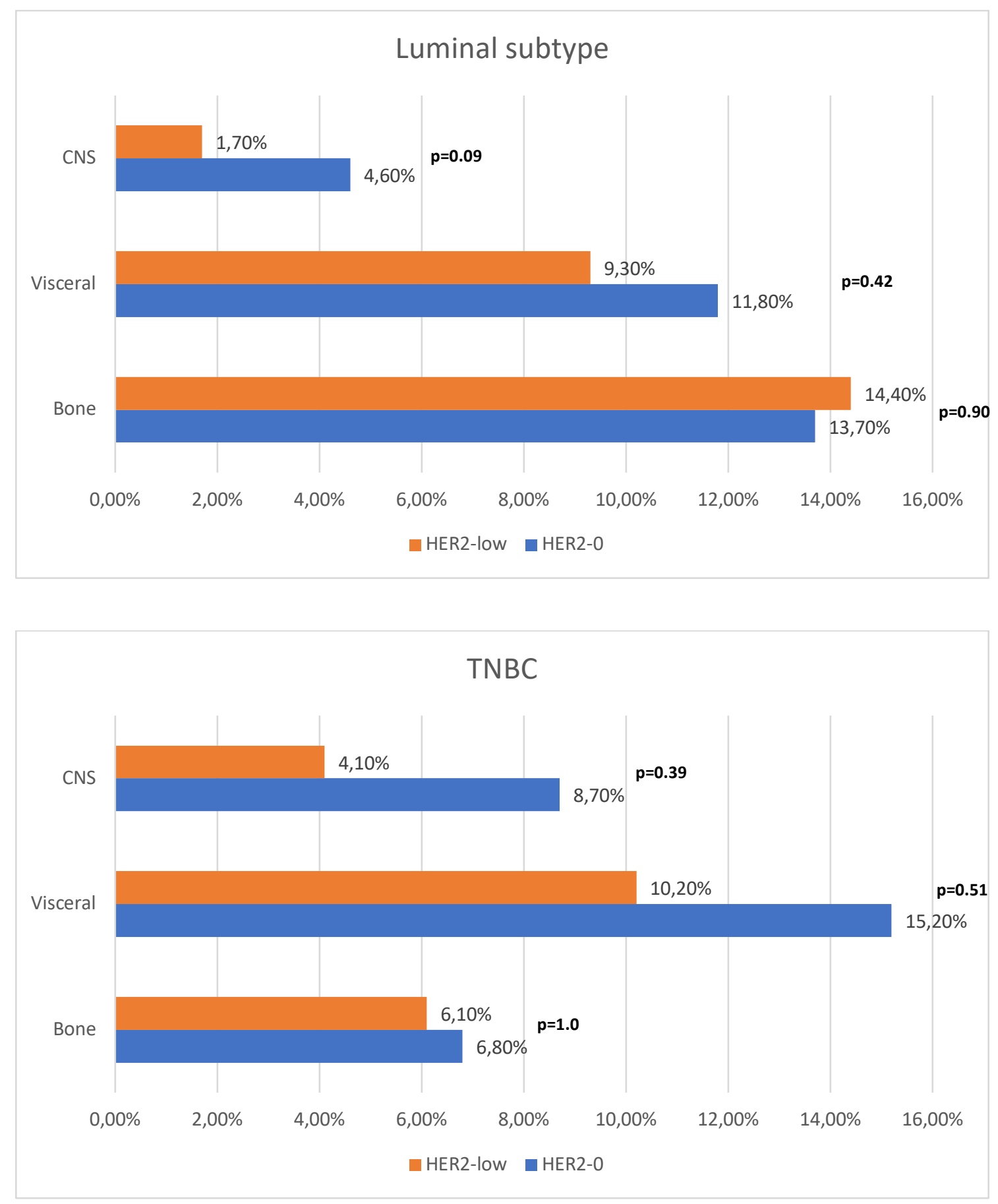

Supplementary figure 3 . Sites of recurrence in in Luminal/HER2-low ( $N=236)$, Luminal/HER2-0 ( $N=306)$ and TNBC/HER2-low ( $N=49)$, TNBC/HER2-0 ( $N=264)$. Frequencies were compared with Fisher's exact test. 


\section{References}

1. Goutsouliak K, Veeraraghavan J, Sethunath V, et al (2020) Towards personalized treatment for early stage HER2-positive breast cancer. Nat Rev Clin Oncol 17:233-250. https://doi.org/10.1038/s41571-019-0299-9

2. Cesca MG, Vian L, Cristóvão-Ferreira S, et al (2020) HER2-positive advanced breast cancer treatment in 2020. Cancer Treat Rev 88:102033. https://doi.org/https://doi.org/10.1016/j.ctrv.2020.102033

3. Slamon DJ, Clark GM, Wong SG, et al (1987) Human breast cancer: correlation of relapse and survival with amplification of the HER-2/neu oncogene. Science (80) 235:177 LP - 182. https://doi.org/10.1126/science.3798106

4. Cameron D, Piccart-Gebhart MJ, Gelber RD, et al (2017) 11 years' follow-up of trastuzumab after adjuvant chemotherapy in HER2-positive early breast cancer: final analysis of the HERceptin Adjuvant (HERA) trial. Lancet 389:1195-1205. https://doi.org/https://doi.org/10.1016/S0140-6736(16)32616-2

5. Delaloge S, Antoine A, Debled M, et al (2020) 279MO Divergent evolution of overall survival across metastatic breast cancer (MBC) subtypes in the nationwide ESME real life cohort 2008-2016. Ann Oncol 31:S352-S353. https://doi.org/10.1016/j.annonc.2020.08.381

6. Gianni L, Lladó A, Bianchi G, et al (2010) Open-Label, Phase II, Multicenter, Randomized Study of the Efficacy and Safety of Two Dose Levels of Pertuzumab, a Human Epidermal Growth Factor Receptor 2 Dimerization Inhibitor, in Patients With Human Epidermal Growth Factor Receptor 2-Negative Metastat. J Clin Oncol 28:1131-1137. https://doi.org/10.1200/JCO.2009.24.1661

7. Mass RD, Press MF, Anderson S, et al (2005) Evaluation of Clinical Outcomes According to HER2 Detection by Fluorescence In Situ Hybridization in Women with Metastatic Breast Cancer Treated with Trastuzumab. Clin Breast Cancer 6:240-246. https://doi.org/10.3816/CBC.2005.n.026

8. Fehrenbacher L, Cecchini RS, Geyer CEJ, et al (2020) NSABP B-47/NRG Oncology Phase III Randomized Trial Comparing Adjuvant Chemotherapy With or Without Trastuzumab in High-Risk Invasive Breast Cancer Negative for HER2 by FISH and With IHC 1+ or 2. J Clin Oncol Off J Am Soc Clin Oncol 38:444-453. https://doi.org/10.1200/JCO.19.01455

9. Modi S, Park H, Murthy RK, et al (2020) Antitumor Activity and Safety of Trastuzumab Deruxtecan in Patients With HER2-Low-Expressing Advanced Breast Cancer: Results From a Phase Ib Study. J Clin Oncol Off J Am Soc Clin Oncol 38:1887-1896. https://doi.org/10.1200/JCO.19.02318

10. Banerji U, van Herpen CML, Saura C, et al (2019) Trastuzumab duocarmazine in locally advanced and metastatic solid tumours and HER2-expressing breast cancer: a phase 1 dose-escalation and dose-expansion study. Lancet Oncol 20:1124-1135. https://doi.org/10.1016/S1470-2045(19)30328-6

11. Tarantino P, Hamilton E, Tolaney SM, et al (2020) HER2-Low Breast Cancer: Pathological and Clinical Landscape. J Clin Oncol Off J Am Soc Clin Oncol 38:1951-1962. https://doi.org/10.1200/JCO.19.02488

12. Wolff AC, Hammond MEH, Allison KH, et al (2018) Human Epidermal Growth Factor Receptor 2 Testing in Breast Cancer: American Society of Clinical Oncology/College of American Pathologists Clinical Practice Guideline Focused 
Update. J Clin Oncol Off J Am Soc Clin Oncol 36:2105-2122.

https://doi.org/10.1200/JCO.2018.77.8738

13. Wolff AC, Hammond MEH, Hicks DG, et al (2013) Recommendations for Human Epidermal Growth Factor Receptor 2 Testing in Breast Cancer: American Society of Clinical Oncology/College of American Pathologists Clinical Practice Guideline Update. J Clin Oncol 31:3997-4013. https://doi.org/10.1200/JCO.2013.50.9984

14. Wolff AC, Hammond MEH, Schwartz JN, et al (2007) American Society of Clinical Oncology/College of American Pathologists guideline recommendations for human epidermal growth factor receptor 2 testing in breast cancer. Arch Pathol Lab Med 131:18-43. https://doi.org/10.1043/1543-

2165(2007)131[18:ASOCCO]2.0.CO;2

15. Schalper KA, Kumar S, Hui P, et al (2013) A Retrospective Population-Based Comparison of HER2 Immunohistochemistry and Fluorescence In Situ Hybridization in Breast Carcinomas: Impact of 2007 American Society of Clinical Oncology/ College of American Pathologists Criteria. Arch Pathol Lab Med 138:213-219. https://doi.org/10.5858/arpa.2012-0617-OA

16. Rossi V, Sarotto I, Maggiorotto F, et al (2012) Moderate immunohistochemical expression of HER-2 (2+) without HER-2 gene amplification is a negative prognostic factor in early breast cancer. Oncologist 17:1418-1425. https://doi.org/10.1634/theoncologist.2012-0194

17. Schettini F, Chic N, Brasó-Maristany F, et al (2021) Clinical, pathological, and PAM50 gene expression features of HER2-low breast cancer. npj Breast Cancer 7:1. https://doi.org/10.1038/s41523-020-00208-2

18. Lambein $K$, Van Bockstal M, Vandemaele L, et al (2013) Distinguishing score 0 from score 1+ in HER2 immunohistochemistry-negative breast cancer: clinical and pathobiological relevance. Am J Clin Pathol 140:561-566. https://doi.org/10.1309/AJCP4A7KTAYHZSOE

19. Eggemann $\mathrm{H}$, Ignatov $\mathrm{T}$, Burger $\mathrm{E}$, et al (2015) Moderate HER2 expression as a prognostic factor in hormone receptor positive breast cancer. Endocr Relat Cancer 22:725-733. https://doi.org/10.1530/ERC-15-0335

20. Reinert T, Sartori GP, Souza AAB, et al (2021) Abstract PS4-22: Prevalence of HER2-low and HER2-zero subgroups and correlation with response to neoadjuvant chemotherapy (NACT) in patients with HER2-negative breast cancer. Cancer Res 81:PS4-22 LP-PS4-22. https://doi.org/10.1158/15387445.SABCS20-PS4-22

21. Eiger D, Agostinetto E, Saúde-Conde R, de Azambuja E (2021) The Exciting New Field of HER2-Low Breast Cancer Treatment. Cancers 13

22. Clifton GT, Hale D, Vreeland TJ, et al (2020) Results of a Randomized Phase IIb Trial of Nelipepimut-S + Trastuzumab versus Trastuzumab to Prevent Recurrences in Patients with High-Risk HER2 Low-Expressing Breast Cancer. Clin Cancer Res 26:2515 LP - 2523. https://doi.org/10.1158/1078-0432.CCR-19-2741

23. Mittendorf EA, Lu B, Melisko M, et al (2019) Efficacy and Safety Analysis of Nelipepimut-S Vaccine to Prevent Breast Cancer Recurrence: A Randomized, Multicenter, Phase III Clinical Trial. Clin Cancer Res 25:4248 LP - 4254. https://doi.org/10.1158/1078-0432.CCR-18-2867

24. Rossi L, McCartney A, Risi E, et al (2019) Cyclin-Dependent Kinase 4/6 Inhibitors in Neoadjuvant Endocrine Therapy of Hormone Receptor-Positive Breast 
Cancer. Clin Breast Cancer 19:392-398.

https://doi.org/10.1016/j.clbc.2019.05.019

25. Schmid P, Cortes J, Pusztai L, et al (2020) Pembrolizumab for Early TripleNegative Breast Cancer. N Engl J Med 382:810-821.

https://doi.org/10.1056/NEJMoa1910549 
Figures

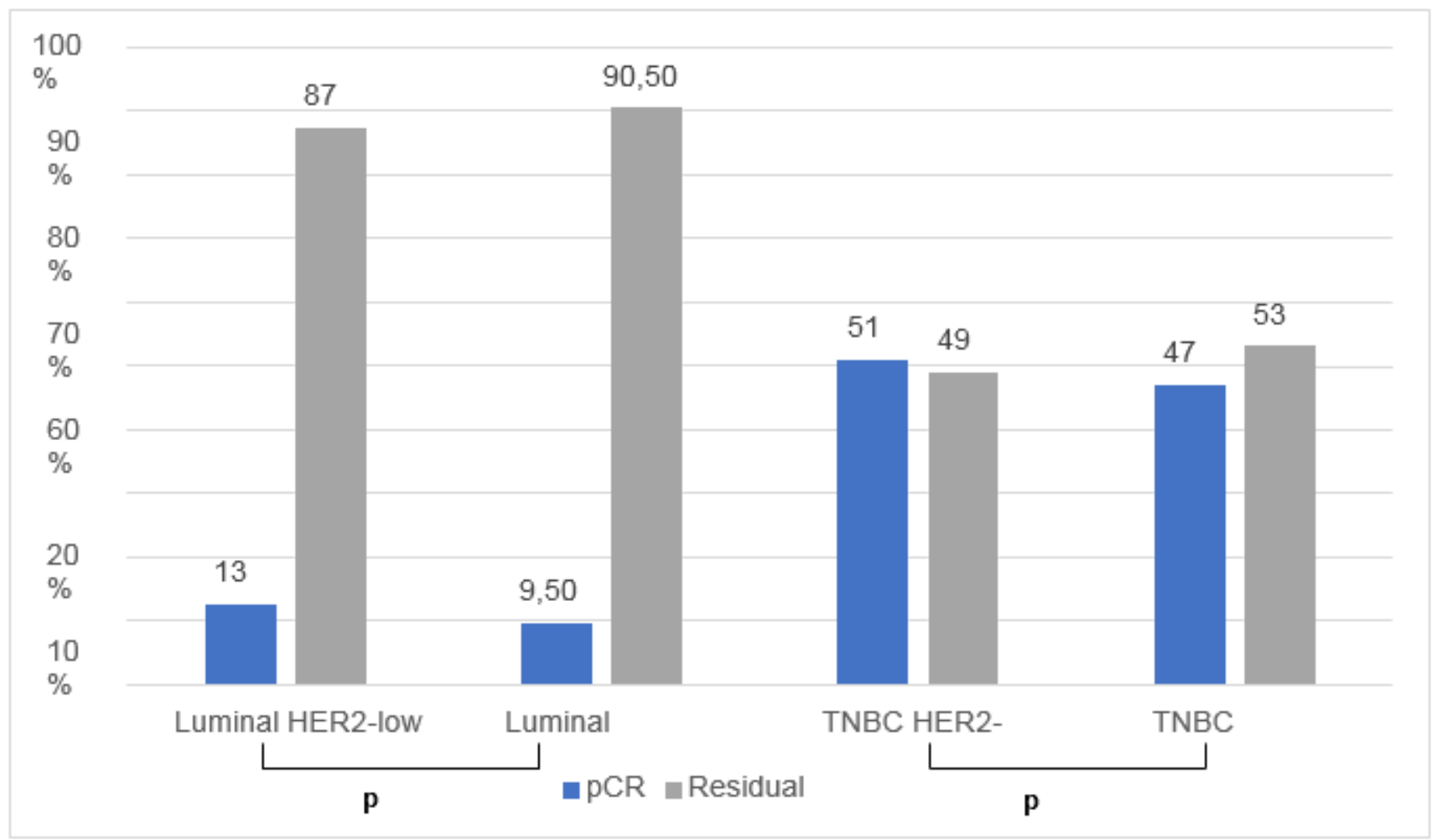

Figure 1

Frequency of pCR in Luminal/HER2-low $(\mathrm{N}=236)$, Luminal/HER2-0 $(\mathrm{N}=306)$ and TNBC/HER2-low $(\mathrm{N}=49)$, TNBC/HER2-0 ( $\mathrm{N}=264)$. pCR was defined as no invasive carcinoma in the breast and in axillary lymph nodes at the time of surgery. Frequencies were compared with Fisher's exact test. 


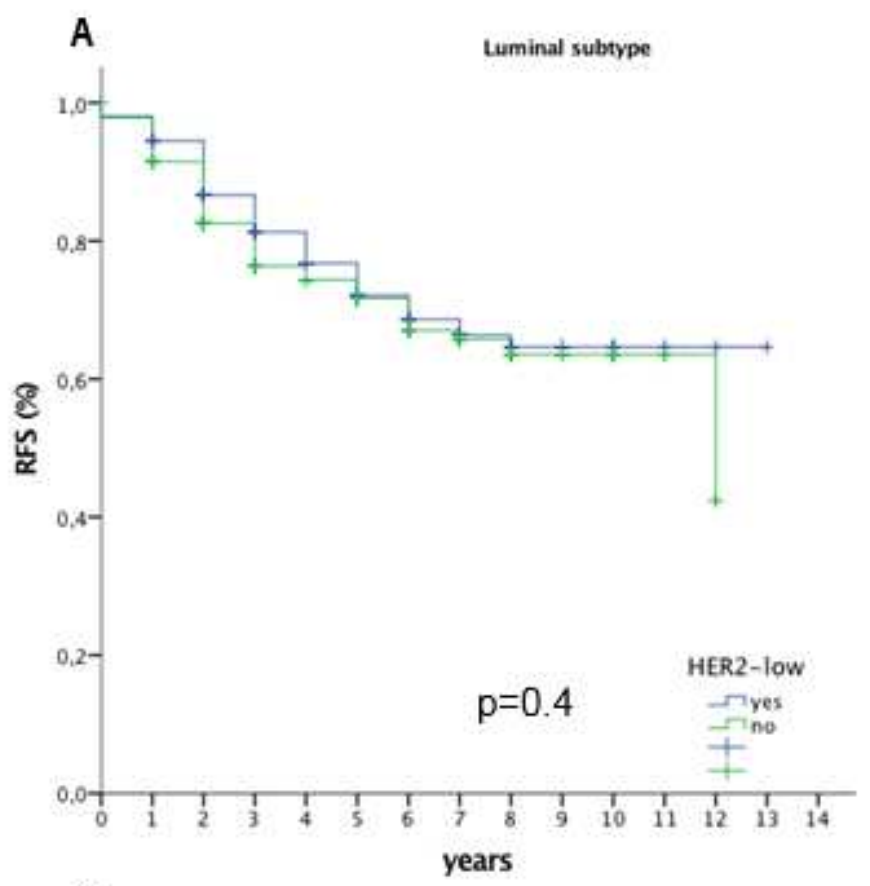

C

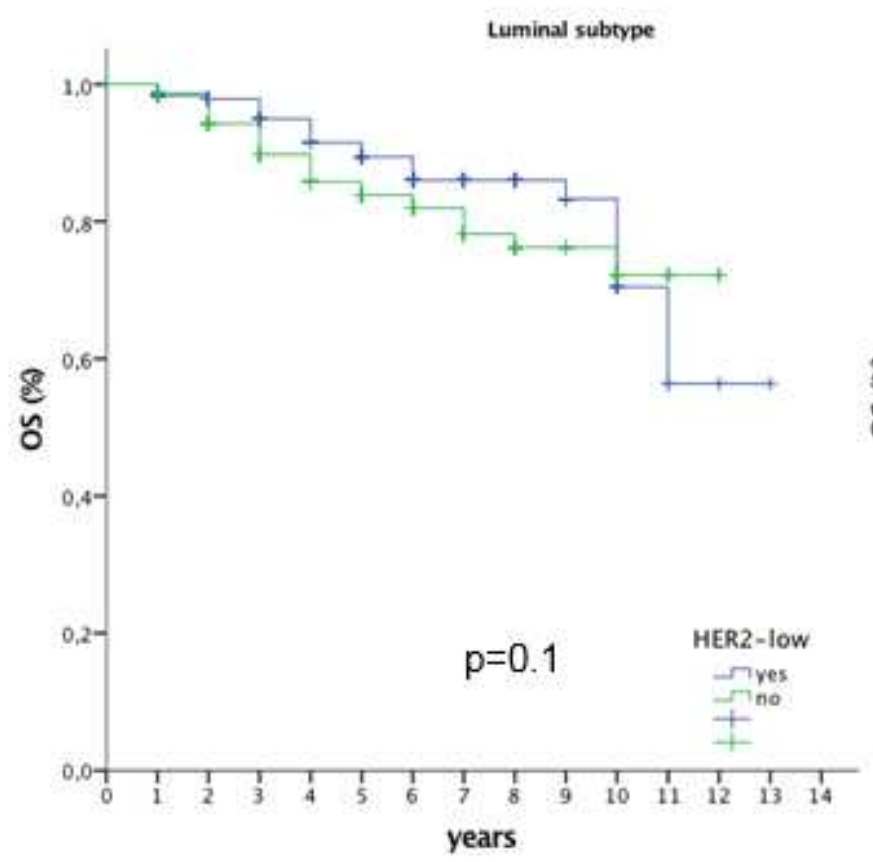

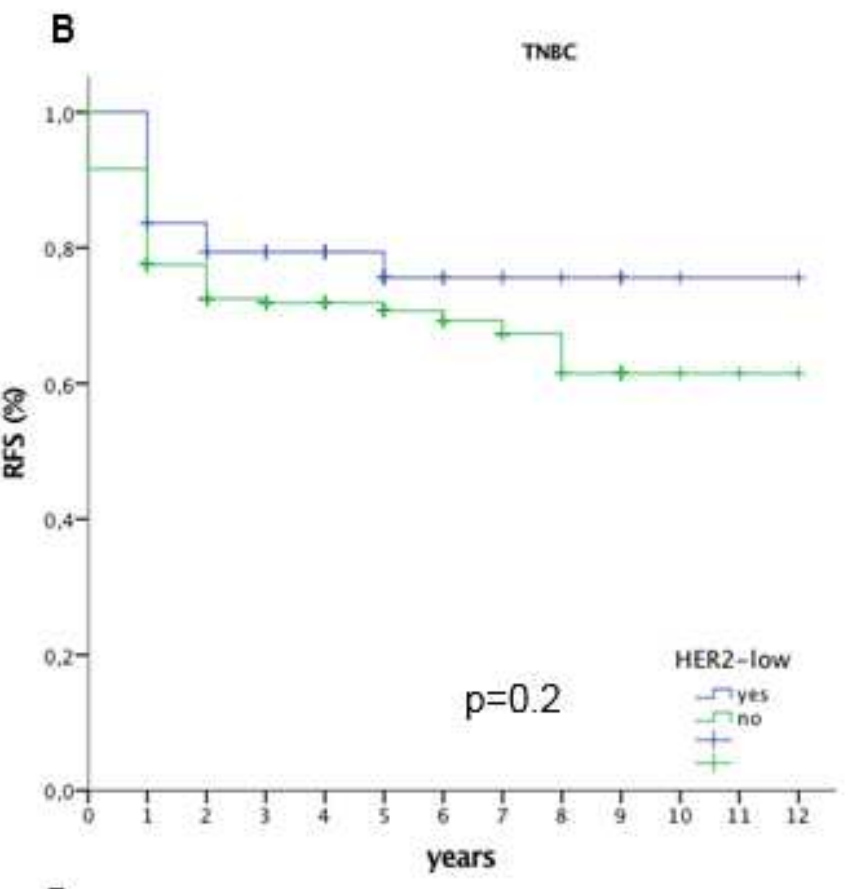

D

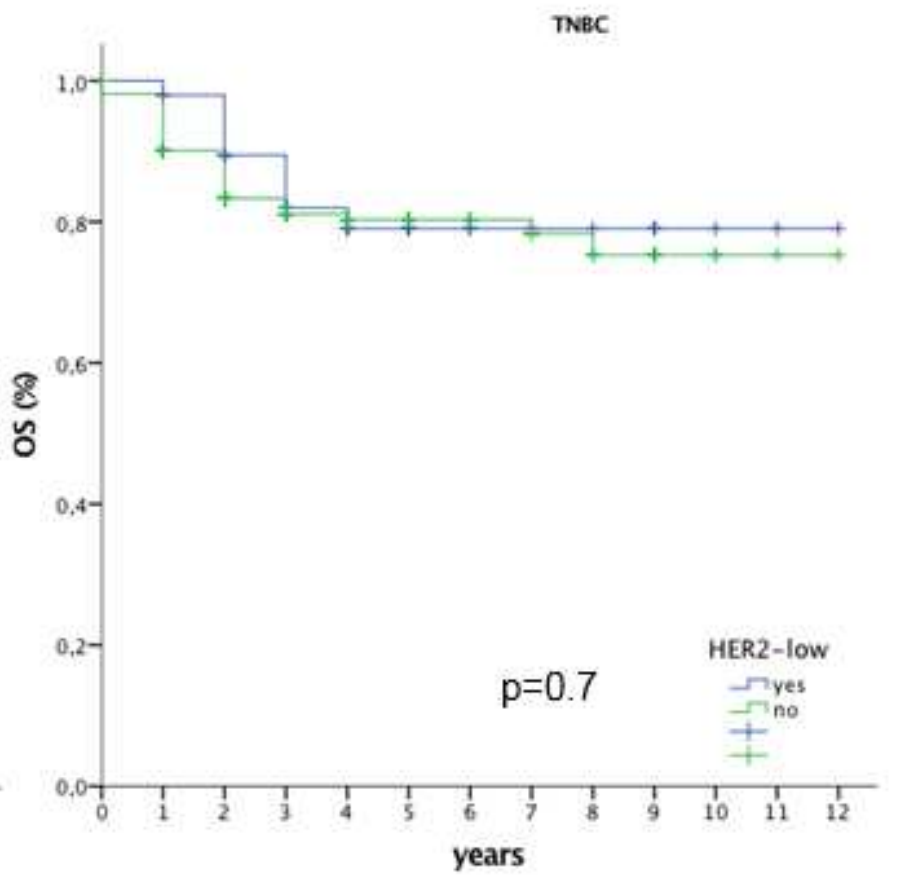

\section{Figure 2}

Relapse-free and overall survival curves according to HER2-low classification among molecular subtypes. $A$ and $B$ show relapse-free survival curves according to molecular subtype (luminal/TNBC) stratified by HER2-low status. $C$ and $D$ show overall survival curves according to molecular subtype (luminal/TNBC) stratified by HER2-low status. Survival curves were estimated with the Kaplan-Meier method and compared with log-rank test. 


\section{Supplementary Files}

This is a list of supplementary files associated with this preprint. Click to download.

- SupplementaryFiguresTables.docx 